\title{
371. Die Anatomie des Trigonum caroticum
}

\author{
C. Reuter, E. Kiffner und A. Valesky \\ Klinik für Chirurgie, Medizinische Hochschule, Ratzeburger Allee 160, D-2400 Lübeck
}

\section{The Anatomy of the Carotid Triangle}

Summary. Thromboendarterectomy of the carotid artery is often performed as a prophylactic operation. Therefore the operative risk is to be minimized. Preoperative evaluation of local operability and application of knowledge of topographic anatomy prevent intraoperative lesions. The variations of the carotid artery and its branches are shown in respect to local operability. The special topographic anatomical relationships between vagal nerve, hypoglossal nerve and glomus caroticum as well as the surgical procedure are presented.

Key words: Carotid artery - Topographic anatomy - Surgical procedure.

Zusammenfassung. Die Desobliteration der A. carotis ist häufig ein propylaktischer Eingriff. Dies beinhaltet die Forderung nach geringstmöglichem Risiko. Die exakte Abklärung der Operabilität sowie Kenntnisse der topograpischen Anatomie sind zur Vermeidung iatrogener Nervenläsionen Voraussetzung. Die Ausstellung zeigt die häufigsten Varianten des Aufzweigungstyps der A. carotis. Außerdem werden die zu berücksichtigenden anatomischen Besonderheiten bei der Hautincision, von Venen, N. vagus, N. hypoglossus und Glomus caroticum dargestellt.

Schliisselwörter: A. carotis - Topographische Anatomie - Chirurgisches Vorgehen.

\section{Die endoskopische Operation in der Rectumhöhle}

\author{
G. Bueß, R. Theiß, F. Hutterer, H. Pichlmaier, C. Pelz, Th. Hohlfeld, S. Said und W. Isselhard \\ Chirurgische Universitätsklinik Köln, Institut für experimentelle Medizin Köln, \\ Joseph-Stelzmann-Straße 9, D-5000 Köln 41
}

\section{Endoscopic Surgery in the Rectum}

Summary. The use of newly designed instruments allows surgical operations in the rectum up to $20 \mathrm{~cm}$ from the dentate line under endoscopic control. The rectum has to be widened by a combination of mechanical and pneumatic dilatation. The stereoscopic oblique-angle instrument is adjusted to the operation-area. We can use up to four instruments simultaneously. After injection of POR (ornipressin) into the mucosa we have excised areas $3 \mathrm{~cm}$ in diameter. The defect is closed by continuous suture.

Key words: Sessile polyp - Small carcinoma of the rectum - Transanal excisions - Endoscopic surgery.

Zusammenfassung. Wir können mit einem neu entwickelten Instrumentarium unter endoskopischer Kontrolle in der Rectumhöhle differenzierte, chirurgische Eingriffe bis in $20 \mathrm{~cm}$ Höhe durchführen. Dazu wird die Rectumhöhle unter kombinierter mechanischer und pneumatischer Dehnung entfaltet und der zu operierende Prozeß mit einer stereoskopischen Winkeloptik eingestellt. Für die Operation können bis zu 4 Instrumente gleichzeitig eingesetzt werden. Wir haben Schleimhautexcisionen von $3 \mathrm{~cm}$ Durchmesser nach POR (Ornipressin)-Unterspritzung vorgenommen und den entstandenen Defekt mit einer fortlaufenden Naht verschlossen.

Schliisselwörter: Breitbasiger Polyp - Kleines Rectumcarcinom - Transanale Abtragung - Endoskopische Chirurgie. 